\title{
Gossip in Evolutionary Perspective
}

\author{
R. I. M. Dunbar \\ University of Liverpool
}

\begin{abstract}
Conversation is a uniquely human phenomenon. Analyses of freely forming conversations indicate that approximately two thirds of conversation time is devoted to social topics, most of which can be given the generic label gossip. This article first explores the origins of gossip as a mechanism for bonding social groups, tracing these origins back to social grooming among primates. It then asks why social gossip in this sense should form so important a component of human interaction and presents evidence to suggest that, aside from servicing social networks, a key function may be related explicitly to controlling free riders. Finally, the author reviews briefly the role of social cognition in facilitating conversations of this kind.
\end{abstract}

For reasons that are not entirely clear, gossip has acquired a decidedly shady reputation. It is seen as malicious, destructive, and largely reprehensible. Describing a person as an "old gossip" implies someone with more time on their hands than they know what to do with, too much hanging over the garden gate waiting for some passerby to pause for idle chat. To engage in gossip is to speak ill of one's fellows, to interfere with the smooth running of the social relationships within which we are all embedded: in a word, to undermine the very fabric of society. Yet, the term gossip itself did not originally have that meaning. It meant simply the activity that one engaged in with one's "godsibs," one's peer group equivalent of godparents: in other words, those with whom one was especially close.

Whether it is in fact the case that tale-telling and tittle-tattle are all that we do with our nearest and dearest is, perhaps, a moot point. I want, instead, to focus on the broader nature of this activity and argue that gossiping (though perhaps not gossip in its contemporary malicious form) is the core of human social relationships, indeed of society itself. Without gossip, there would be no society. In short, gossip is what makes human society as we know it possible.

Correspondence concerning this article should be addressed to R. I. M. Dunbar, Economic and Social Science Research Council Research Centre in Economic Learning and Social Evolution, School of Biological Sciences, University of Liverpool, Crown Street, Liverpool L69 7ZB, England. E-mail: rimd@liv.ac.uk
To be able to make this claim, I need first to step back in evolutionary time to what we might see as the ancestral state from which modern humans sprang. This is the nature of social relationships that pertain among our primate cousins. I then argue that language evolved as a mechanism for bonding large social groups, and that it does so precisely because it allows us to exchange information about the state of our social networks. In this context, the problem of free riders (those who take the benefits of sociality without paying the costs) is a central issue for which gossip provides a particularly powerful mechanism of control.

\section{Origins}

Humans are members of the order primates, a large and diverse group of mammals of very ancient lineage. We belong to that subgroup of primates known as the catarrhines, the Old World monkeys and apes. We share with these monkeys and apes a deep sociality that is predicated on relatively (by comparison with other mammals and birds) advanced forms of social cognition. This is represented in humans by such phenomena as theory of mind (Astington, 1993; Whiten, 1991; Wimmer \& Perner, 1983) and more advanced forms of the intentional stance (Kinderman, Dunbar, \& Bentall, 1998), which appear to be unique to humans (Call \& Tomasello, 1999; Tomasello \& Call, 1998). (The "intentional stance" is the phenomenon of interpreting behavior in terms of the belief states of the mind that is behind the behavior, something that seems to be especially charac- 
teristic of humans [Dennett, 1983].) Social cognition in these respects is based on the reflexively hierarchical phenomenon of mind-reading, the ability reflected in the claim that $I$ suppose that you believe that I want you to think that $X$ is the case.

The extent to which other monkeys and apes share these particular capacities is not germane to the thesis of this article. The issue is more that Old World monkey and ape social relationships seem to be underpinned by relatively sophisticated forms of social cognition that find their fullest expression in the reflexive multilevel hierarchy of intentionality that we find in living humans. I return to this particular issue in the final section; first, I simply want to establish why it is that the capacity to exchange information (i.e., language) evolved at all in humans.

Anthropoid primate societies (and especially those of the Old World monkeys and apes) are characterized by an intensity of sociality that is not as conspicuous in other species. These species are deeply social; they are social in a sense that is all too readily apparent to anyone who has ever taken the trouble to spend any time observing their behavior. This intensity of sociality depends on two key phenomena. One is the ability to understand something of the workings of another's mind. By this, I do not mean to suggest that monkeys and apes understand that other individuals have minds in the way that we humans take this for granted. Theirs is more the ethologist's skill at reading behavior (Cheney \& Seyfarth, 1990). In effect, they are good at understanding correlations in behavior: that individuals behave in certain consistent ways that can vary widely from one individual to another, that others' behavior can be deliberately manipulated to one's own advantage.

The second feature underpinning primate sociality is the use of trust and (in a weak sense) obligation to ensure that relationships work effectively and do the job they are intended to do. For monkeys and apes, predators are the single most important factor influencing group size: As a species' ecological habits expose it to increased risk of attack by predators, so its group size must be proportionately larger to protect its members (for reviews, see Dunbar, 1988, 1996a). Although protection against predators can be seen as clearly advantageous for primates, it is important not to lose sight of the fact that sociality has costs as well as benefits.
Living together exposes animals to a number of stresses that include disturbances to feeding when one animal displaces another from a feeding site, harassment by more dominant individuals, and the generally disruptive effects that arise from the fact that animals in social groups are obliged to coordinate their behavior in ways that are not always ideal for each individual. Because the consequences of predation are so final for the individual, the costs of sociality must be held in check to minimize their impact; otherwise, the centrifugal forces of individuals' selfish demands will rapidly and inevitably result in the dispersal of the group. Sociality, in short, demands compromise on one's personal, short-term objectives so that one gains in the longer term through a greatly reduced risk of falling victim to a predator.

The primate solution to this problem (essentially the need to balance short-term interests against the longer term gains to be had through group living) is the formation of alliances. Often, these alliances are deeply rooted in matrilineal relationships (mothers and daughters, sisters). These relationships work (and are crucial in the life histories of the animals themselves) because they involve a strong element of trust and commitment. An alliance member can be relied on to come to one's aid at the crucial moment when one is under attack. That sense of obligation is created through social grooming. We do not really understand how grooming makes this possible, though we do know that grooming is extremely effective at releasing endorphins (endogenous opiates produced naturally by the brain as part of the body's pain control system; Keverne, Martensz, \& Tuite, 1989). The flood of opiates triggered by being groomed (and perhaps even by the act of grooming itself) generates a sense of relaxation (grooming lowers heart rate, reduces signs of nervousness such as scratching, and can so relax the groomee that it may even fall asleep; Goosen, 1981).

We are ourselves familiar with these effects, because we show a striking preference for resorting to old-fashioned primate grooming in our more intimate relationships; here language is an inadequate means for communicating deep inner feelings (and especially emotions), and we often resort to physical contact forms of communication (rubbing, stroking, patting, and petting) that are extremely effective at triggering 
the release of endorphins. As the endorphins triggered by these behaviors begin to flood the body, we experience a rising sense of warmth, a feeling of peace with the world, of well-being toward those with whom we share such experiences of intimacy. The effect is instantaneous and direct: The physical stimulation of touch tells us more about the inner feelings of the "groomer," and in a more direct way, than any words could possibly do.

The key issue at this point is that the amount of grooming that primates do is directly related to the size of the groups they live in, apparently because the effectiveness with which an alliance works is a simple function of the amount of time devoted to grooming by its members. Although, within a species, alliance size does not vary greatly as group size increases, the effectiveness with which the alliance must work does correlate with group size. As group size increases, individuals are subjected to increasing levels of ecological competition (local food sources are exhausted more quickly, forcing animals to search more widely for food) and reproductive stress (harassment by others is sufficient to destabilize a female's menstrual hormone cycles, leading to anovulatory or infertile cycles; for a review, see Dunbar, 1988). The more stress imposed on the individual, the more effective its alliances must be to buffer it against these stresses. Because there must be an upper limit on the amount of time that can be devoted to social grooming, there will inevitably be an upper limit on the size of the group that can be bonded by this mechanism (Dunbar, 1998a). In actuality, of course, grooming time is constrained by the fact that animals have to forage for food. In practice, it seems that there is an upper limit on the amount of time that any given group of primates devote to grooming, and this is about $20 \%$ of the total waking time in each day. Given the nature of the activity, this is, of course, a very substantial amount of time: Roughly one fifth of the day is being devoted to social investment.

More important, however, this figure is equivalent to a group size of about 80 animals (Dunbar, 1992, 1998a). (Note that these values correspond to the mean group sizes for individual species, not the maximum possible group sizes that we might observe.) The problem for modern humans is that we have a natural group size of about 150 individuals (roughly equiva- lent to the number of individuals one knows personally; Dunbar, 1993; Hill \& Dunbar, in press). At some point in our evolutionary history, hominid groups began to push against the ceiling on group size. The only way they could have broken through this ceiling so as to live in groups larger than about 80 individuals was to find an alternative mechanism for bonding in which the available social time was used more efficiently.

Language appears to serve that function perfectly, precisely because it allows a significant increase in the size of the interaction group (Dunbar, 1993, 1996b). Grooming is very much a one-on-one activity (it still is, even for us), whereas conversation group sizes typically contain up to four individuals (invariably one speaker and three listeners; Dunbar, Duncan, \& Nettle, 1995). In addition, speaking is something that we can do simultaneously with most other activities. As a result, we can "time share" more effectively to cram more into what limited time we do have available. Significantly perhaps, it turns out, from a sample of people's time budgets drawn from a wide range of cultures around the world, that the average percentage of time humans spend in social interaction (mainly conversation, of course) is $20 \%$ (Dunbar, 1998b). In other words, our social time allocation is at the upper limit of that seen in primates; we simply use the time more efficiently because language allows us to do so.

Note that neither of these benefits requires more than speech; content is not explicitly required, because all that is necessary is to convey a message of social commitment ("I consider it more important standing here talking to you than being over there with [... anyone else]"). However, one further key feature of language is particularly important to the bonding of our unusually large social groups, namely the fact that language allows us to exchange information. That, after all, is what language itself is basically designed to do. Its role in social bonding is that it allows us to keep track of what is going on within our social networks, as well as using it to service those relationships. As important as the latter function is, it is in many ways the first that is especially important. Lacking language, monkeys and apes are constrained in what they can know about their networks. They know only what they see. We are similarly limited when it comes to first-hand knowledge. 
But language allows us to seek out what has been going on behind our backs. Indeed, we can even be proactive about it and tell our friends and relations what we have seen when we think it might be in their interests to know.

Let me summarize the argument thus far. Primates live in groups to protect themselves against predation, and group size increases as predation risk increases. But group living comes at a cost in terms of the stresses it imposes on the individual. Monkeys and apes solve this problem by forming alliances that buffer them against these costs, thus facilitating the cohesion of large social groups. These alliances are bonded through social grooming (a process that is mediated pharmacologically through the release of endorphins), but the cohesion of large social groups also depends on a unique form of cognitive engagement that allows animals to understand and exploit the mental states of other individuals. Sociality in primates is thus dependent on a two-pronged process. One involves heavy cognitive demands, in which any specieslevel increases in group size that are required to allow animals to exploit more predator-risky habitats are necessarily predicated on corresponding increases in brain size (and explicitly frontal lobe size) to handle the social cognitive demands of managing proportionately more relationships. The other is a more ancient process based on the exploitation of grooming-based pharmacological mechanisms that facilitate bonding so as to allow the processes of mutual support on which group cohesion ultimately depends to work effectively.

The evolutionary sequence here is this: Exploitation of more predator-risky habitats requires an increase in group size; to make this possible, it is necessary both to evolve the cognitive machinery to underpin the management of the social relationships involved (essentially a larger neocortex) and to invest more time in the necessary bonding processes. Humans represent the most extreme point in this sequence within the primates because hominid evolution has been characterized by the exploitation of increasingly open terrestrial habitats, both of these features being associated with increased predation risk. It may be that in the later stages of hominid (human?) evolution, the risk of predation by other humans became more important than the risks of predation by more conventional predators (Johnson \& Earle, 1987), but this does not obviate the fundamental issue that risk of death from predators (of whatever kind) is the principal factor favoring increases in group size. Language became part of this story because, at some point in hominid evolutionary history, the group size required exceeded that which could be bonded through social grooming alone; the constraint in this context was the fact that the time investment required by grooming is ultimately limited by the demands of foraging. Language enabled hominids to break through that particular glass ceiling because it allows time to be used more effectively than is possible with grooming: Speech allows us both to interact with a number of individuals simultaneously (grooming is a strictly one-on-one activity) and to exchange information about the state of our social network (lacking language, monkeys and apes are limited in their knowledge of their network by what they themselves see). In the next section, I elaborate on this theme by arguing that the exchange of social information (i.e., gossip) has been crucial to our ability as a species to evolve large social groups.

\section{The Functions of Gossip}

A strong case can, then, be made for the suggestion that language evolved to facilitate the bonding of large social groups. It achieves this mainly because it allows us to increase the size of our broadcast network (the number of people with whom we can communicate directly and indirectly) and because it allows us to exchange information about changes that occur within our social networks. We can catch up on news of Uncle Joe and Aunt Jane, discover that Susan and Bill have parted company (and so avoid an embarrassing faux pas that might otherwise have upset what had once been a good relationship), and learn about new additions to the family.

But language allows us to do much more than this. We can use language in at least four other ways (there may be others too, of course, but these will at least serve to illustrate the point I want to make here). One is to seek advice or to discuss hypothetical situations: "How would you behave if ...?" A second is to provide us with a kind of policing function to control those who fail to abide by the formal and informal agreements that underpin society. (I have more to say about this particular issue in the next 
section.) The third possibility is that we can use language to advertise ourselves: Look at all my wonderful qualities as a potential mate or ally. Finally, in a variant on the last, we can use language to deceive: to tell others what we think it would help us for them to know, as when Iago poisoned Othello's mind against Desdemona.

It is perhaps important to appreciate that, although we can use language in all of these ways and possibly more, all of them are really derivative of the fact that language evolved to allow us to bond large social groups. None of these additional functions would really be relevant (or so intrusive) if we did not first live in large groups. In contrast, without language as a means of exchanging information about the social network, large groups could not be kept together as viable, coherent social entities. So although we may use language to deceive or police, the possibility of being able to use it in this way would not exist without there being large groups in the first place.

However, we should first ask what evidence there is that language is used in these various ways. In part, this really concerns the distinction between social and technical uses of language. Language is concerned with the exchange of information; that, after all, is what it (or, at least, grammar) is mainly designed to do. However, linguists and those in most other disciplines interested in language have traditionally assumed that the information to be exchanged is factual knowledge about the world; in other words, language evolved to allow our ancestors to exchange information about aspects of the physical world in which they lived. "This is how you make a good spear.... If you throw your spear this way, you are more likely to kill the prey. ... I just saw some bison down by the lake as I was passing, so let's go hunt them.... Never swim in the river because crocodiles [or dangerous spirits] live there and will grab you!"

Of course, we can do amazing technical things only because we have language: Without language, Pythagoras would not have been able to explain his theorem, Newton would never have been able to expound on the nature of gravity, and probably none of their fellow men and women would have been the slightest bit interested in their rather exotic theories because they would not have seen the relevance of them. Who cares if the square on the hypotenuse is equal to the sum of the squares on the other two sides? Should I care why apples fall to earth so long as I can pick them up? It is enough that we know that they do! The reality, however, is that language allows us to stand, as Newton so memorably put it, on the shoulders of the giants who went before us; language, and nothing else, has made the spectactular growth of knowledge in the last few centuries possible.

This technological or economic view of what language is used for has so deeply colored our perception of the function of language that it has led us to see any other uses of language as trivial and largely driven by the boredom of idleness. Thus has gossip acquired its ill repute, for it is seen as mere time wasting when we could be doing something really useful with our time such as talking about jet engines or the technical details of how to earn more money by making our businesses more efficient. The real issue here, then, is which of these two broad uses of language is the chicken, and which is the egg? In other words, did language evolve to facilitate the exchange of technological information (with gossip as an idle by-product), or did it evolve to facilitate the exchange of social information (with the exchange of technological information as a useful by-product)?

The issue here is a purely empirical one: How $d o$ we use language? What $d o$ we actually talk about? My colleagues and I have carried out a number of observational studies of the content of conversations. All of these studies have been undertaken in public places, and we simply note the topic under discussion in free-running conversations at 30-s intervals. To ease the process of classification for the observer and avoid problems of unnecessary intrusion, we use only broadly defined categories (e.g., social, politics, sports, music and culture, and technical). The "social" category covers anything that has to do with explicitly social activities, personal relationships, and personal likes or dislikes. Our aim here is to try to gain a basic description of how people use language in relaxed everyday situations.

It is worth pointing out that almost all of the work carried out hitherto on conversations has been subject to one or both of two key distortions, namely the use of artificially convened groups or a focus on workplace environments. These circumstances introduce distortions because the situations are unnatural (despite the length of time we might be said to spend in our 
places of work). Such distortions have not really been an issue in previous studies, because these investigations have focused on aspects of conversational behavior other than topic of conversation (e.g., the mechanics of conversation, how conversations are paced, and the cues used in signaling switching of roles; Beattie, 1983; Coates, 1993). However, these artificialities introduce serious distortions when our concern is to assess whether or not conversations are used to service existing relationships, because most such situations either do not involve individuals who have relationships or focus on contexts in which relationships are of a very specific and short-term nature (e.g., workplace relationships). It would be futile (and bizarre) to try to study how (or, worse still, whether) language is used to service social relationships by ensuring that there were no relationships to be serviced. In our design, participants can talk about relationships if they feel them to be important, but not if they do not wish to do so.

The results we have obtained from a series of studies in different locations have been very consistent: Social topics (what I here define broadly as "gossip") account for approximately $65 \%$ of speaking time, with only limited variation due to age or gender (Dunbar, Duncan, \& Marriott, 1997; Seepersand, 1999). All of the other topics summed together account for only about one third of conversation time. Thus, these findings suggest that naturally occurring conversations are dominated by social topics. Although non-European conversations have yet to be extensively studied in this way, there is nothing in the ethnographic literature (or, indeed, my own experience of traditional African societies) to suggest that members of other cultures behave differently. The one study that did involve an analysis of conversation content in a non-European population (in this case, Zinacantan Indians in Mexico) showed that $78 \%$ of topics recorded in 1,754 conversations were concerned with social issues (Haviland, 1977). (This study did not sample conversation topics at a set time interval, but rather counted all of the different topics that were talked about in a substantial number of conversations.) However, less systematic evidence suggests that social gossip has always been important: In medieval Europe, for example, a person's fama (or reputation) was highly dependent on what others said about him or her and was thus a source of considerable personal angst as well as something to be manipulated by others in conversations (Fenster \& Smail, 2003). Indeed, Emler (1990) has argued that reputation management is a major objective of gossip even in modern societies.

If language is principally used for social exchanges in everyday life, then the obvious next question is how language is used to facilitate social relationships in groups. I noted earlier that there are at least four different ways in which language might contribute to this: keeping track of other individuals in the network; advertising one's own advantages as a friend, ally, or mate (or perhaps the disadvantages of potential rivals); seeking advice on personal problems; and, finally, policing free riders. We attempted to assess the relative balance among these four possible functions by analyzing in greater detail social topics of conversation. The results of this more detailed study of 30 freely formed conversations revealed, somewhat to our surprise, that most social conversation time in our sample was devoted to the first two topics (in about equal quantities). Soliciting advice and policing free riders each accounted for only about 5\% of total social conversation time (Dunbar et al., 1997).

I express surprise here because we had anticipated that the free rider problem (as described subsequently) would be so intrusive in everyday life that it would result in constant need of the policing function. That policing free riders seemed to be so rare a topic of conversation could be due to the particular circumstances under which we made our observations, which took place in open public environments (cafes, restaurants, bars, and trains). It may well be that people are reluctant to discuss more intimate topics in public, where they may be overheard; such conversations may be confined to more private venues. If we were to sample conversations across the full range of an individual's time, it might be that more conversation time would be devoted to these two topics. Alternatively, it may be that these kinds of topics rear their ugly head only at relatively infrequent intervals. The need to discuss such topics may be rare, but when they occur they may, of course, be disproportionately important in the lives of the individuals concerned because the consequences of being exploited by a free rider are especially damaging. 
In summary, then, language in freely forming natural conversations is principally used for the exchange of social information. That such topics are so overwhelmingly important to us suggests that this is a primary function of language. It is interesting in this context to note that most social topics focus on events in one's social network and using language to promote one's particular social opportunities. Seeking advice and engaging in policing functions seem to be less common. Notwithstanding the results just described, the issue of free riders is one of fundamental importance in human societies. In the next section, I spell out the problem in more detail and argue that the issue of abusive gossip is directly derivative of this issue.

\section{The Free Rider Problem}

One reason why gossip has acquired its negative connotations is that conversation is sometimes genuinely used to comment on the behavior of, or caste aspersions on the character of, those of whom we disapprove. One reason why we might have a predisposition to behave in this way is that free riders are extremely destructive for societies based on a social compact.

I suggested earlier that the principal evolutionary innovation of the primates has been their ability to exploit social solutions to the everyday problems of survival and successful reproduction. Such solutions are dependent on an implicit social contract whereby each individual agrees to forgo some of his or her immediate interests to gain in the long term through collaborative solutions to the ecological problems that threaten everyday survival. However, social arrangements of this kind are extremely susceptible to free riders, those who take the benefits of sociality but decline to pay all of the costs. Detailed mathematical modeling of virtual societies based on these kinds of social contracts suggests that free riders become increasingly more intrusive as social group sizes increase or society becomes more dispersed, principally because under each of these conditions free riders benefit from having large numbers of naive individuals whom they can exploit (Enquist \& Leimar, 1993). In this context, "naive" means individuals who have no knowledge of the free rider's behavior and thus interact with the free rider in an open and trusting way, thereby exposing themselves to exploitation.
The central problem for highly social species is that it would be extremely inefficient to treat everyone we come across with the level of suspicion and caution that would be required to ensure that we are never exploited. To do so would waste time and resources that could more profitably be spent pursuing genuinely beneficial social opportunities. Indeed, in the final analysis, sociality itself would break down because no one would be willing to cooperate, and hence the whole point of living together and sharing social activities would evaporate. If sociality is to provide us with the benefits it is designed to provide, then we have to be prepared to take risks and trust the bona fides of those with whom we interact. It is this trustingness that provides the crucial loophole that free riders exploit. In a word, free riding works as a strategy mainly because most members of the community take each other at face value.

As trusting as we often are, it is clear that free riding is a serious social problem. We operate many low-level defenses against free riders on a daily basis. We search each other's faces and expressions for cues of honesty and dishonesty. Folk wisdom and our proverbs are littered with allusions to cues of honesty and dishonesty (e.g., "Never trust a man who cannot look you in the eye"). We exert a level of caution against strangers, testing their intentions first with small gifts before we offer them our lives and souls; conversely, when anxious to solicit large-scale favors, we offer substantial gifts up front to declare the honesty of our intentions. Nettle and Dunbar (1997) used a modeling approach to show that dialects are ideally suited to act as a mechanism for controlling free riders, providing that they change rapidly from one generation to the next. Because they are difficult to acquire if they are not learned early in life, dialects allow community members to identify those who are likely to share a common history of obligation (and hence to be trustworthy reciprocators).

But free riders need not always be strangers who hove into view over the horizon from far away. They may just as easily be members of our community. Some may be individuals who have always cut their relationships with a very fine knife, ensuring that they never give too much away; others may be individuals who, perhaps because of a change of circumstances, calculatedly seize an opportunity to exploit a situation to their advantage. We are always at 
risk from the individual whose natural character is not to give too much away or who is willing to exploit friends and acquaintances when an opportunity arises.

Indeed, in many ways, our attitude toward kin and close friends tends to be much more exploitative than we might typically suppose: We tend to take them for granted, make more social effort on behalf of acquaintances or those we see less often than those we know best. One example of this is the fact that we tend to greet distant acquaintances more effusively than we do those whom we see more often (Kendon, 1973), even though the latter are likely to be more important to us in terms of support and social environment. Although this may partly reflect the need to emphasize a nonthreatening attitude toward those with whom we are less familiar, it also implies that we are much more willing to trade on the good nature of those with whom we have well-established relationships.

The control of free riders is largely dependent on memory of past events (occasions on which they have cheated us or reneged on implicit contracts) and an appreciation of how they might afflict others (which depends on sophisticated cognitive abilities such as theory of mind). However, although we may appreciate the consequences for others of a free rider's behavior, until and unless we communicate our concerns there is no social component to these concerns. Only when we advise potential victims of the danger they face are we exercising any kind of social censure. Language is clearly central to that process.

Enquist and Leimar (1993) modeled this situation using a form of evolutionary analysis based on the well-known social psychological paradigm of the "prisoner's dilemma." They sought to identify the evolutionary-stable strategy (defined in evolutionary biology as the strategy that cannot be outcompeted by any alternative mutant strategy) that maximized the genetic fitness of individual members of a community when these individuals had to collaborate with each other to reproduce successfully. They showed that, under a range of realistic assumptions, free riders who engaged in collaboration to accept resource donations but later failed to repay these "gifts" would invariably outreproduce conventional collaborators who behaved honestly. In a social environment that initially consisted of honest reciprocators and a single "mutant" free rider, the reciprocators would, within a very small number of generations, be driven to extinction by the free riders. However, if community members were able to pass on even a modest amount of information about the behavior of individual free riders, the free riders' freedom of movement would be greatly curtailed, and honest reciprocators would be able to retain a dominating presence in the population. Enquist and Leimar (1993) explicitly referred to this process of information exchange as "gossip."

Although, in our studies of freely forming conversations, we found that gossip of this censorious type was relatively rare, it may nonetheless be that the handful of cases involving this kind of behavior are disproportionately important in terms of their consequences for the recipients' ability to avoid exploitation in the future. This is clearly much less easy to study, because it involves a long-term future and would thus require the longitudinal study of individual participants over many months, perhaps even years. Such studies could (and, indeed, should!) be done, and they might best be done with some kind of diary methodology; however, they would inevitably be laborious and very time consuming.

The potentially important role of verbal censure in controlling free riders has been studied in a series of small group experiments conducted by Orstrom and colleagues (Orstrom, Gardner, \& Walker, 1994). In these experiments, participants were invited to make financial investments in one of two markets. Participants worked at individual computer terminals, but the terminals were linked so that each participant could see all of the other bids being made in the successive rounds of the game (but not who was making them). The markets were structured in such a way that whereas one yielded a constant return, the other yielded returns that were an exponential function of the number of investors. Thus, participants playing in these markets soon learned that the optimal payout was gained only if everyone invested in the second market. However, if not everyone invested in this way, the payout was poorer than could be gained by investing in the first market. With the odds stacked to provide a temptation to go it alone, the experiments yielded results in which the mean payout was typically as low as $20 \%$ of that which could have been achieved 
had everyone followed the collaborative strategy, even though everyone's bids could clearly be seen.

Orstrom et al. (1994) found that introducing even a single refreshment break in which participants could meet significantly reduced the number of defections during subsequent rounds, thus raising the mean payout to approximately $80 \%$ of the maximum. In these refreshment breaks, individuals were allowed to discuss the experiment, but they still did not know which participants had been responsible for the defections in previous rounds. Thus, simply allowing participants free reign to comment on the defections or to exhort the other participants to toe the line had a dramatic effect in reducing the temptation to defect (in other words, act as a free rider). In a subsequent experiment, participants were allowed to punish defectors during the game (by requesting that a still anonymous defector be fined by the experimenter), and this resulted in a further dramatic improvement in performance (and thus an increase in mean payout). However, by comparison with the opportunity to exhort free riders during the refreshment break, the additional gain provided by the opportunity to punish was modest.

These simple experiments suggest that even the opportunity to comment on the behavior of free riders can exert a significant effect on free riders' willingness to break ranks on implicit or explicit social contracts. This provides confirmation that we are sensitive to others' opinions of us, such that other members of society can exploit this sensitivity to keep us in line. This does not, of course, mean that such a strategy will work for everyone, but it seems that it works sufficiently often to ensure that only a relatively small number of individuals try to exploit the loopholes in the fabric of the social world.

\section{Cognitive Underpinnings of Gossip}

In the earlier sections, I alluded a number of times to the role of cognition (and particularly the higher levels of social cognition) in managing primate and human social relationships. I want to end by exploring the relevance of this issue to gossip in a little more detail.

Social cognition (typically exemplified by theory of mind) plays an important role in human relationships. The ability to see the world from another person's point of view is a fundamental prerequisite for successful social interaction. In the present context, it is important for two reasons.

One is that it allows us to recognize that someone else might be at risk of exploitation by a free rider even though we ourselves are not (because we have identified the free rider or taken steps to neutralize his or her ability to exploit us). Naive individuals lack that foresight, and we are able to recognize this only because we can see the world from their point of view and sympathize with their potential future predicament. Conversely, having a theory of mind (or, better still, more advanced levels of cognition) allows free riders to act more effectively: Being able to put themselves in other people's mental shoes gives them a significant advantage in identifying (and hence exploiting) potential victims.

The other respect in which social cognition is necessary is that language itself probably requires at least second-order intentionality and, possibly, higher levels (Dunbar, 1998b). When we engage in conversation, we can use words in their literal sense, or we can use them metaphorically. Human conversation is littered with metaphor and is typically telegraphic in style. In part, that may reflect a desire for economy, but equally it reflects a desire to be less than direct in what we have to say. We speak opaquely in riddles to ensure that our real meaning is obscured from those we would rather did not discern it. This places a considerable load on both speaker and listener. The latter in particular has to work very hard trying to figure out just what it is that the speaker is trying to tell him or her. This use of metaphor may be especially valuable in allowing us to comment on a certain member of society in a manner only specific individuals will understand.

Humans achieve second-order intentionality (formal theory of mind) at approximately 4 years of age (Astington, 1993), but normal adults are capable of negotiating fourth- or perhaps even fifth-order intentionality (Kinderman et al., 1998; Stiller \& Dunbar, 2003). Such levels of sophistication probably play an important role in allowing us to dig deeply into the intentions and honesty of those with whom we interact on a daily basis. They certainly influence the size of our social network (Stiller \& Dunbar, 2003). However, we still have a very 
poor understanding of the role of advanced social cognition in the everyday social interactions of normal adults. So far as I know, the Kinderman et al. (1998) study and two unpublished studies (Stiller \& Dunbar, 2003; Swarbrick, 2000) are the only studies that have attempted to explore intentionality beyond the second level (theory of mind).

\section{Conclusion}

I have argued that gossip, in the broad sense of conversation about social and personal topics, is a fundamental prerequisite of the human condition. Were we not able to engage in discussions of these issues, we would not be able to sustain the kinds of societies that we do. Gossip in this broad sense plays a number of different roles in the maintenance of socially functional groups through time; although simple social bonding is perhaps the single most important of these roles (and was perhaps the original impetus to the evolution of language), language permits other social functions. Of these, the exchange of information on free riders has undoubtedly become important in the large dispersed societies of modern humans. In some respects, its development may be seen as a natural outgrowth of our social brain, because it exploits the intense interest that we naturally have in the doings of others. That it can be carried to extremes may be a matter for regret, but this should not distract us from the central issue that gossip (in its broadest sense) is the central plank on which human sociality is founded. In reality, the cognitive demands of gossip are the very reason why such large brains evolved in the human lineage.

\section{References}

Astington, J. W. (1993). The child's discovery of the mind. Cambridge, England: Cambridge University Press.

Beattie, G. (1983). Talk: An analysis of speech and non-verbal behavior in conversation. Milton Keynes, England: Open University Press.

Call, J., \& Tomasello, M. (1999). A nonverbal theory of mind test: The performance of children and apes. Child Development, 70, 381-395.

Cheney, D., \& Seyfarth, R. (1990). How monkeys see the world. Chicago: University of Chicago Press.

Coates, J. (1993). Men, women and language. New York: Longman.
Dennett, D. (1983). Intentional systems in cognitive ethology: The 'Panglossian paradigm' defended. Behavioral and Brain Sciences, 6, 343-390.

Dunbar, R. I. M. (1988). Primate social systems. London: Chapman \& Hall.

Dunbar, R. I. M. (1992). Neocortex size as a constraint on group size in primates. Journal of $\mathrm{Hu}$ man Evolution, 20, 469-493.

Dunbar, R. I. M. (1993). Coevolution of neocortical size, group size and language in humans. Behavioral and Brain Sciences, 16, 681-735.

Dunbar, R. I. M. (1996a). Determinants of group size in primates: A general model. In J. Maynard Smith, G. Runciman, \& R. I. M. Dunbar (Eds.), Evolution of culture and language in primates and humans (pp. 33-57). Oxford, England: Oxford University Press.

Dunbar, R. I. M. (1996b). Grooming, gossip and the evolution of language. Cambridge, MA: Harvard University Press.

Dunbar, R. I. M. (1998a). The social brain hypothesis. Evolutionary Anthropology, 6, 178-190.

Dunbar, R. I. M. (1998b). Theory of mind and the evolution of language. In J. Hurford, M. StuddertKennedy, \& C. Knight (Eds.), Approaches to the evolution of language (pp. 92-110). Cambridge, England: Cambridge University Press.

Dunbar, R. I. M., Duncan, N. D. C., \& Marriott, A. (1997). Human conversational behavior. Human Nature, 8, 231-246.

Dunbar, R. I. M., Duncan, N. D. C., \& Nettle, D. (1995). Size and structure of freely forming conversational groups. Human Nature, 6, 67-78.

Emler, N. (1990). A social psychology of reputation. European Journal of Social Psychology, 1, 171193.

Enquist, M., \& Leimar, O. (1993). The evolution of cooperation in mobile organisms. Animal Behaviour, 45, 747-757.

Fenster, T., \& Smail, D. L. (2003). Fama: The politics of talk and reputation in medieval Europe. Ithaca, NY: Cornell University Press.

Goosen, C. (1981). On the function of allogrooming in Old World monkeys. In A. B. Chiarelli \& R. S. Corruccini (Eds.), Primate behavior and sociobiology (pp. 110-120). Berlin: Springer.

Haviland, J. B. (1977). Gossip, reputation and knowledge in Zinacantan. Chicago: University of Chicago Press.

Hill, R. A., \& Dunbar, R. I. M. (in press). Human social networks. Human Nature.

Johnson, A. W., \& Earle, T. (1987). The evolution of human societies: From foraging groups to agrarian state. Stanford, CA: Stanford University Press.

Kendon, A. (1973). A description of some human greetings. In J. H. Crook (Ed.), Comparative ecology and behavior of primates (pp. 591-668). London: Academic Press. 
Keverne, E. B., Martensz, N. D., \& Tuite, B. (1989). Beta-endorphin concentrations in cerebrospinal fluid of monkeys are influenced by grooming relationships. Psychoneuroendrocrinology, 14, 155-161.

Kinderman, P., Dunbar, R. I. M., \& Bentall, R. (1998). Theory-of-mind deficits and causal attributions. British Journal of Psychology, 89, 191-204.

Nettle, D., \& Dunbar, R. I. M. (1997). Social markers and the evolution of reciprocal exchange. Current Anthropology, 38, 93-98.

Orstrom, E., Gardner, R., \& Walker, J. (1994). Rules, games and common-pool resources. Ann Arbor: University of Michigan Press.

Seepersand, F. (1999). Laughter and language in evolution. Unpublished master's thesis, University of Liverpool, Liverpool, England.

Stiller, J., \& Dunbar, R. I. M. (2003). Perspective taking and social network size in humans. Manuscript submitted for publication.
Swarbrick, R. (2000). A social cognitive model of paranoid delusions. Unpublished doctoral dissertation, University of Manchester, Manchester, England.

Tomasello, M., \& Call, J. (1998). Primate social cognition. Oxford, England: Oxford University Press.

Whiten, A. (Ed.). (1991). Natural theories of mind. Oxford, England: Blackwell.

Wimmer, H., \& Perner, J. (1983). Beliefs about beliefs: Representation and constraining function of wrong beliefs in young children's understanding of deception. Cognition, 13, 103-128.

Received June 2, 2003

Revision received July 9, 2003

Accepted July 9, 2003

\section{Wanted: Old APA Journals!}

APA is continuing its efforts to digitize older journal issues for the PsycARTICLES database. Thanks to many generous donors, we have made great strides, but we still need many issues, particularly those published in the 1950 s and earlier.

If you have a collection of older journals and are interested in making a donation, please e-mail journals@apa.org or visit http://www.apa.org/journals/donations.html for an up-to-date list of the issues we are seeking. 